\title{
Efecto del consumo de Moringa oleífera sobre parámetros productivos y toxicológicos en pollos de engorda
}

\author{
Martha K. Fuentes Esparza ${ }^{\text {a }}$ \\ Teódulo Quezada Tristán ${ }^{\text {a* }}$ \\ Salvador H. Guzmán Maldonado ${ }^{\text {b }}$ \\ Arturo G. Valdivia-Flores ${ }^{\text {a }}$ \\ Raúl Ortíz-Martínez ${ }^{\text {a }}$
}

a Universidad Autónoma de Aguascalientes. Centro de Ciencias Agropecuarias, Departamento de Clínica Veterinaria, Av. Universidad N 904, 20131 Aguascalientes, Aguascalientes, México.

${ }^{\mathrm{b}}$ Instituto Nacional de Investigaciones Forestales, Agrícolas y Pecuarias. Laboratorio de Alimentos Funcionales, Guanajuato. México.

*Autor de correspondencia: tquezada@ correo.uaa.mx

\section{Resumen:}

Es necesario explorar fuentes de proteína alterna para adicionar a la alimentación de aves en regiones rurales. Son contados los estudios realizados en moringa para este propósito. Se determinó el análisis proximal, el contenido de Fe, Ca y taninos a la harina de hoja de moringa. Se evaluó el efecto del consumo de una dieta con $10 \%$ de harina sobre cuatro parámetros productivos y seis serológicos (dos proteínas y cuatro enzimas) en pollos de engorda Ross-308. También se realizaron estudios histopatológicos del hígado y riñón de los pollos. La hoja de moringa presentó $33.4 \%$ de proteína y un alto contenido de hierro $(19.7 \mathrm{mg} / 100 \mathrm{~g})$ y calcio $(2593.3 \mathrm{mg} / 100 \mathrm{~g})$ así como bajo contenido de taninos $(24.4 \mathrm{mg}$ EC/100 g). Se observó una reducción del 12 y $20 \%$ en la ganancia en peso y en el índice de productividad, respectivamente, en los pollos a los que se les adicionó moringa. No se observaron diferencias entre el grupo control y el adicionado con moringa en el contenido de proteínas y la actividad de la alanino aminotransferasa y la aspartato aminotransferasa, pero si en la albúmina, la fosfatasa alcalina y la gama galutamil transpeptidasa, que 
sugiere cierto daño hepático y renal. Estos resultados no se observaron en los estudios histológicos. Se sugiere seguir realizando estudios con niveles de complementación menor con la harina de moringa para comparar los efectos encontrados en el presente estudio y así poder definir su uso potencial.

Palabras clave: Pollos de engorda, Moringa, Productividad, Serología, Histopatología.

Recibido: $14 / 08 / 2017$

Aceptado: 20/02/2018

La avicultura a nivel comercial es una actividad importante en México ${ }^{(1)}$. Para alimentar a las aves se ha utilizado la pasta de algodón, cacahuate, cártamo, ajonjolí y coco como fuente de proteína y otros nutrientes, pero son deficientes en lisina. Por el contrario, la pasta de soya es la fuente proteica que presenta un balance óptimo de aminoácidos para la alimentación animal ${ }^{(2)}$. Por otro lado, la avicultura incluida la engorda de pollo de traspatio, es una actividad importante en las comunidades rurales del país. No solo provee a las familias de carne y huevo, también de recursos por la venta de los excedentes ${ }^{(3)}$. De lo anterior se desprende la necesidad de explorar fuentes de proteína de especies locales que sean accesibles y que puedan ser añadidos a los alimentos como el maíz, restos de alimentos y otros, que en las regiones rurales destinan a las aves.

Una especie que ha sido estudiada como fuente de proteína para animales ${ }^{(4,5)}$ y humanos $^{(6)}$ es la moringa (Moringa oleífera). Esta especie fue introducida en México de la India y Paquistán $^{(7)}$. La hoja de la moringa contiene 20 a $30 \%$ de proteína, 5.0 a $7.5 \%$ grasa y 25 a $31 \%$ fibra $^{(8,9)}$. Además, es una buena fuente de hierro, calcio y vitamina $\mathrm{C}^{(9)}$. Debido a la presencia de otros fitoquímicos que contiene, la hoja de moringa también ha sido recomendada para tratar ulceras gastrointestinales ${ }^{(10)}$ bajar el colesterol y como fuente de antioxidantes $^{(11)}$.

En relación con el uso de la moringa en la alimentación animal, la hoja ha sido utilizada para alimentar conejos ${ }^{(12)}$, cabras $^{(5)}$, ovejas ${ }^{(13)}$ y vacas $^{(14)}$, entre otras especies. Basados en la revisión bibliográfica realizada se identificaron pocos estudios donde se haya utilizado hoja de moringa para alimentar pollos de engorda. En este sentido, se han realizado trabajos para evaluar el efecto de la hoja de moringa en la morfología del intestino y la condición de otros órganos internos ${ }^{(15,16)}$, el contenido de ácidos grasos en la pechuga ${ }^{(17)}$, en la fuerza de la tibia y su contenido de minerales $^{(18)}$, el peso, la hematología y respuesta inmune $^{(4,19)}$. De estos reportes solo dos investigaciones han realizado estudios para evaluar el efecto de la hoja de moringa en la ganancia en el peso ${ }^{(4,19)}$; sin embargo, uno de ellos fue realizado con Moringa stenopetala ${ }^{(4)}$ que es una especie diferente a la 
Moringa oleífera, utilizada en este estudio, aunque son del mismo género.

El objetivo de este trabajo fue evaluar el efecto de harina de hoja de moringa sobre parámetros productivos y efectos tóxicos potenciales en pollos de engorda. La caracterización nutrimental y el contenido de vitamina $\mathrm{C}$ y taninos condensados, de la hoja de moringa.

Se cosecharon hojas de moringa de árboles de dos años de una huerta ubicada en el municipio de Gasca en Guanajuato, México. Al momento de la cosecha las hojas se enjuagaron con una solución de hipoclorito de sodio al $0.1 \%$ y luego con agua destilada estéril, para lavar y desinfectar y se secaron a la sombra. Las hojas secas se molieron con un molino de martillo (2.0-3.0 mm de tamizaje) y se almacenaron a $-20{ }^{\circ} \mathrm{C}$ hasta su análisis y uso en las dietas de los pollos. Por otro lado, se adquirieron 180 pollos de engorda, mixtos, de un día de edad de la línea ROSS-308 de $39 \pm 1.5 \mathrm{~g}$ de peso.

El estudio con los pollos se realizó en la Unidad de aves del Área Pecuaria de la Posta Zootécnica de la Universidad Autónoma de Aguascalientes, ubicada en el municipio de Jesús María, Aguascalientes. Los pollos se seleccionaron completamente al azar para formar dos grupos con tres repeticiones de 30 aves cada uno. Los pollos se alimentaron con alimento convencional (T1) y con alimento convencional al que se le añadió $10 \%$ de harina de hoja de moringa (HM). Ambas dietas fueron iso-protéicas e iso-energéticas ${ }^{(20)}$ (Cuadro 1). El alimento y el agua se proporcionaron ad libitum. El tiempo del experimento fue de 42 días.

Cuadro 1: Ingredientes y su composición nutricional de las dietas experimentales

\begin{tabular}{|c|c|c|c|c|}
\hline \multirow[b]{3}{*}{ Ingredientes $(\mathrm{g} / \mathrm{kg})$} & & \multicolumn{3}{|c|}{$\begin{array}{c}\text { Harina de hoja de Moringa } \\
\text { oleifera }(\%)\end{array}$} \\
\hline & $\mathbf{0}$ & 10 & $\mathbf{0}$ & 10 \\
\hline & \multicolumn{2}{|c|}{ Pollos de 1-21 días } & \multicolumn{2}{|c|}{ Pollos de 22-42 días } \\
\hline Sorgo $(8.5 \%)$ & 600 & 560 & 685 & 640 \\
\hline Pasta de soya ( $46 \%)$ & 335 & 280 & 250 & 200 \\
\hline Harina de moringa $(33.4 \%)$ & 0.0 & 100 & 0.0 & 100 \\
\hline Aceite vegetal & 25 & 20 & 25 & 20 \\
\hline Micro pollo eng $1^{\mathrm{a}}$ & 40 & 40 & 0.0 & 0.0 \\
\hline Micro pollo eng $2^{\mathrm{b}}$ & 0.0 & 0.0 & 40 & 40 \\
\hline
\end{tabular}


Valores calculados ${ }^{\mathrm{c}}$

Energía metabólica/ave, kcal/kg

$\begin{array}{cccc}3150 & 3150 & 3200 & 3200 \\ 21.0 & 21.0 & 18.0 & 18.0 \\ 0.52 & 0.54 & 0.48 & 0.50 \\ 0.81 & 0.83 & 0.75 & 0.75 \\ 0.73 & 0.75 & 0.66 & 0.68 \\ 0.23 & 0.24 & 0.20 & 0.21 \\ 0.13 & 0.14 & 0.11 & 0.12 \\ 4.0 & 4.4 & 3.5 & 4.0 \\ 0.884 & 0.902 & 0.824 & 0.411 \\ 0.213 & 0.213 & 0.205 & 0.205 \\ 1.236 & 1.263 & 1.115 & 1.139 \\ 1.137 & 1.163 & 1.029 & 1.050 \\ 0.350 & 0.451 & 0.311 & 0.824\end{array}$

Proteína cruda, \%

Lisina total, \%

Lisina digestible, $\%$

Metionina digestible, $\%$

Metionina+cisteína digestible, \%

Treonina digestible, $\%$

Triptófano digestible, $\%$

Arginina digestible, \%

Calcio, \%

Fósforo disponible, \%

$\mathrm{Na}, \%$

$0.350 \quad 0.451$

0.311

0.824

Fibra cruda, \%

${ }^{\mathrm{a}}$ Complemento alimenticio iniciación (g/kg de producto): L-lisina HCl, 12.6; Metionina, 56.6; Cloruro de Colina al $60 \%$ (Nacional), 10.2; Ortofosfato al $21 \%$, 274; sulfato de cobre al $25 \%$, 10; Sal molida roche, 50; Aceite mineral petroblanc 90 NF, 10; Bacitracina B.M.D.110, 10; Pasta de soya, 169.4; Bicarbonato de sodio, 40; Ronozyme VP, 7.6; Sacox, 10; Calcio M-20, 268. V/inic pollo de engorda plus NF/3*Ton, 60; Vip/Prem/A/Prem Lucantin amarillo al 10, 7.5; Invivo/P/AYC/Prem Ronozyme Hiphos GT/1*Ton, 4

${ }^{\mathrm{b}}$ Complemento Mineral, Vitamínico y de Aminoácidos (kg de producto): Vit A 10,000,000 UI; Vit D3 3,000,000 UI, Vit E 7,000 UI; Vit K 1.5 g; Roboflavina, 4.4 g; Vit B12,15 mg; Ácido pantoténico, 5.5 g; Niacina, $25 \mathrm{~g}$; Biotina, $25 \mathrm{mg}$; Tiamina, $1 \mathrm{~g}$; Ácido Fólico, $0.5 \mathrm{~g}$; Hierro, $32.5 \mathrm{~g}$; Manganeso, $50 \mathrm{~g}$; Zinc, 50 g; Cobre, 4.5 g; Selenio, 150 mg; Yodo, 450 mg; Colina, 150 g; Antioxidante, 50 g. Excipiente: $3 \mathrm{Kg}$.

${ }^{\mathrm{a}}$ Complemento alimenticio finalización (g/kg de producto): L-lisina $\mathrm{HCl}, 25$; L-Treonina, 1.8; Metionina, 64.8; Cloruro de Colina al $60 \%$ (Nacional), 10.2; Ortofosfato al $21 \%$, 186; sulfato de cobre al 25\%, 10; Sal molida roche, 50; Aceite mineral petroblanc 90 NF, 10; Bacitracina B.M.D.110, 10; Pasta de soya, 235.6; Bicarbonato de sodio, 30; Ronozyme VP, 5; Sacox, 11; Progen 20, 5; Calcio M-20, 244. V/inic pollo de engorda plus NF/3*Ton, 64; Vip/Prem/A/Prem Lucantin amarillo al 10, 37.5.

${ }^{\mathrm{b}}$ Complemento Mineral, Vitamínico y de Aminoácidos (Kg de producto): Vit A 10,000,000 UI; Vit D3 3,000,000 UI, Vit E 7,000 UI; Vit K 1.5 g; Roboflavina, 4.4 g; Vit B12,15 mg; Ácido pantoténico, 5.5 g; Niacina, 25 g; Biotina, 25 mg; Tiamina, 1 g; Ácido Fólico, 0.5 g; Hierro, 32.5 g; Manganeso, 50 g; Zinc, $50 \mathrm{~g}$; Cobre, $4.5 \mathrm{~g}$; Selenio, $150 \mathrm{mg}$; Yodo, $450 \mathrm{mg}$; Colina, $150 \mathrm{~g}$; Antioxidante, $50 \mathrm{~g}$.

${ }^{\mathrm{c}}$ Calculado de acuerdo con NRC (1994).

El análisis proximal de la HM se realizó con métodos aprobados para proteína (960.52), fibra $(991.43 \mathrm{G}, \mathrm{H})$, grasa $(920.85)$ y ceniza $(923.03)^{(21)}$. El contenido de carbohidratos se obtuvo por diferencia porcentual con el contenido de los análisis anteriores. El contenido de hierro y calcio se determinó por espectrometría de absorción atómica ${ }^{(22)}$ y la vitamina C por cromatografía líquida de alta resolución (HPLC) ${ }^{(23)}$. También se determinó el contenido de fenoles totales ${ }^{(24)}$, y de taninos condensados ${ }^{(25)}$.

Se registró cada día el alimento proporcionado y el consumido. Al final del experimento se calcularon el peso promedio (PP), la ganancia diaria de peso (GDP), el índice de conversión (IC) y el índice de productividad (IP) ${ }^{(26)}$. 
A los 42 días del experimento se tomaron muestras sanguíneas para la cuantificación de las proteínas totales (PT) y la albúmina (ALB); también se determinaron las enzimas séricas alanino amino transferesa (ALT), aspartato amino transferasa (AST), fosfatasa alcalina (FA) y gama glutamil transpeptidasa $(\mathrm{GGT})^{(27)}$.

Se seleccionaron tres pollos de cada una de las tres repeticiones de los dos grupos originalmente formados para obtener una muestra sanguínea; se sacrificaron por dislocación cervical con base en la guía para el manejo ético y sacrifico humanitario de la Universidad Autónoma de Aguascalientes. Se tomaron muestras de $1 \mathrm{~cm}^{3}$ de hígado y riñón para el estudio histopatológico ${ }^{(28)}$. En el hígado se evaluaron la condición de la histo-arquitectura del parénquima hepático, la presencia o ausencia de células de inflamación, el espacio de Möll, el espacio de Disse, los sinusoides hepáticos y los conductos biliares. En el hepatocito se evaluó la relación núcleo-citoplasma, características del núcleo celular (picnosis, cariolisis y cariorrexis) y la presencia o ausencia de vacuolas lipídicas en el citoplasma.

Los datos del experimento con pollos se sometieron a un análisis de varianza (ANDEVA) y prueba $\mathrm{T}$ de Student con mediciones repetidas y con una $P<0.05$, en un paquete estadístico Statistical Analysis System (SAS, 2001) ${ }^{(29)}$.

La harina de la hoja de moringa utilizada en el presente trabajo presentó mayor contenido de proteína (33.4\%) que los reportados para esta especie $(20.0 \text { a } 29.0 \%)^{(8,9,30)}$ (Cuadro 2). El mayor contenido de proteína observado en las muestras de Guanajuato probablemente se deba a que se trata de otro tipo de moringa o al efecto de la localidad ${ }^{(9)}$. El resto de los compuestos del análisis proximal estuvo dentro del rango reportado en la literatura ${ }^{(8,9,30)}$. Se sabe que la moringa es una buena fuente de hierro y calcio (Ca $2.6 \mathrm{~g}$ y Fe $19 \mathrm{mg} / 100 \mathrm{~g})^{(9)}$, lo cual concuerda con los resultados que se muestran en el Cuadro $2^{(31)}$. En consecuencia, la moringa podría aportar un nivel importante de calcio, al momento de formular un alimento para animales al que se le añada moringa. Por otro lado, se ha reportado que la presencia de hierro en el alimento de pollos promueve no solo la síntesis, también el metabolismo de la vitamina $\mathrm{C}^{(32)}$. En este sentido, el alto contenido de hierro en la moringa junto con la presencia de vitamina $\mathrm{C}$ puede coadyuvar en este fenómeno. 
Cuadro 2: Análisis proximal (\%), contenido de minerales y vitamina C (mg/100 g, MS) y taninos condensados (mg EC/100 g, MS) en hoja de moringa

\begin{tabular}{ll}
\hline Compuesto & Contenido \\
\hline Análisis químico proximal (\%) & \\
Proteína & $33.4 \pm 0.72$ \\
Fibra & $8.8 \pm 0.70$ \\
Grasa & $8.1 \pm 0.41$ \\
Ceniza & $2.3 \pm 0.46$ \\
Carbohidratos & $47.4 \pm 0.52$ \\
Minerales (mg/100 g MS) & \\
Hierro & $19.7 \pm 1.07$ \\
Calcio & $2593.3 \pm 121$ \\
Vitamina C & $63.5 \pm 1.63$ \\
Taninos (mg EC/100 g, MS) & $24.4 \pm 0.92$ \\
\hline
\end{tabular}

$\mathrm{EC}=$ equivalentes de catequina, $\mathrm{MS}=$ materia seca.

Se ha reportado que el uso de sorgo con alto contenido de taninos $(1,360 \mathrm{mg} / 100 \mathrm{~g})$ reduce la ganancia en peso, el consumo de alimento y la absorción de hierro y calcio, entre otros minerales, en pollos de engorda ${ }^{(33)}$. Con base en el muy bajo contenido de taninos que presentó la harina de moringa aquí analizada, se puede concluir que el consumo de moringa utilizado en el presente trabajo no presentó ningún efecto anti nutrimental atribuido a este compuesto fenólico.

Los pollos alimentados con el $10 \%$ de la harina de moringa presentaron $414 \mathrm{~g}$ de peso menos que el grupo testigo (Figura 1A). Esta disminución es el reflejo de la menor cantidad de alimento consumido por los pollos con moringa como se muestra en el Cuadro 3 , donde se puede observar que los consumos promedio de alimento para cada una de las repeticiones de cada tratamiento fue en total de $123.35 \mathrm{~kg}$ para el grupo testigo y 107.19 $\mathrm{kg}$ para el grupo de HM, habiendo una diferencia en el consumo de $16.16 \mathrm{~kg}$ del grupo HM $(P<0.05)$, lo que se pudiera atribuir al incremento de la fibra en la dieta, contrario a lo reportado en pollos con alimento adicionado con HM (5 a $14 \%$ ) con Moringa stenopetala $^{(4)}$. Sin embargo, los pollos a los que se les añadió del 5 al $11 \%$ de $M$. stenopetala no presentaron ninguna diferencia en el peso comparados con el testigo ${ }^{(4)}$; la diferencia observada entre este trabajo y lo que se reporta aquí puede ser atribuido a que se trata de dos especies diferentes. La diferencia en el peso promedio también fue un reflejo en los resultados observados de la ganancia diaria de peso a lo largo del estudio (Figura 1B) En ningún momento este parámetro fue mayor en los pollos con moringa comparado con el grupo testigo. 
Figura 1: A) Peso promedio a los 42 días (g); B) Ganancia diaria de peso (g/día); C) Índice de conversión (consumo de alimento/peso); D) Índice de productividad (ganancia diaria de peso) (viabilidad)/(CAx10) en pollos alimentados con moringa

A)

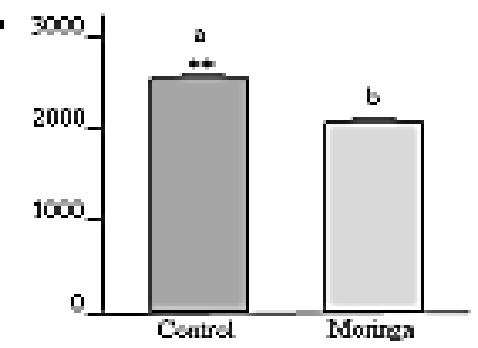

B)
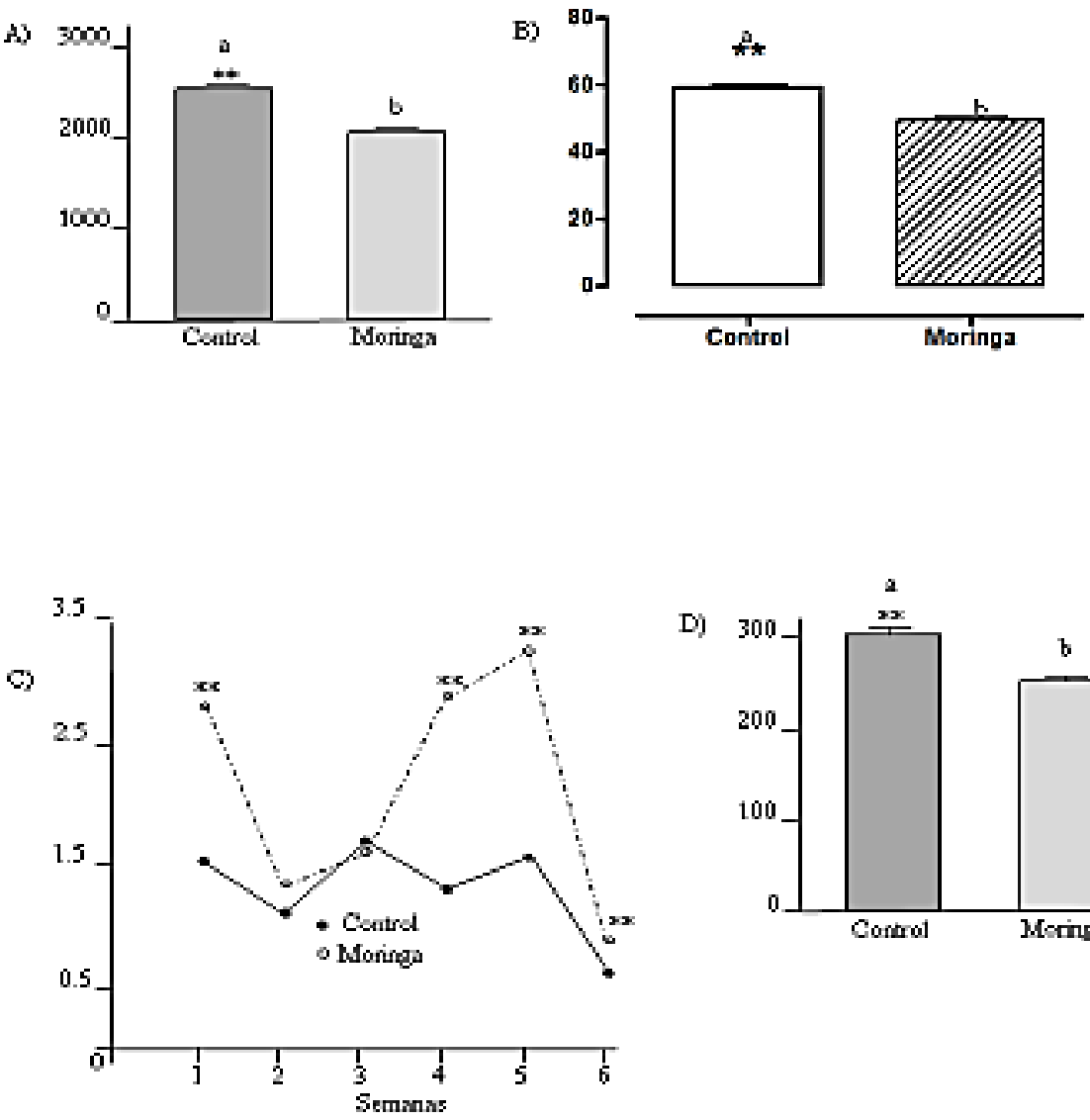

D)

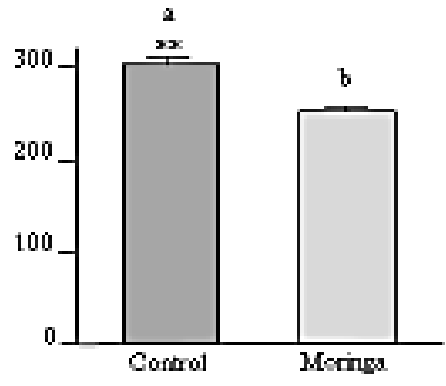

Los asteriscos indican diferencia significativa (T-Student, $P<0.05, \mathrm{n}=63$ ).

Cuadro 3: Consumo promedio de alimento $(\mathrm{kg})$ en el grupo de pollos control y pollos que consumieron HM $(\mathrm{n}=30)$

\begin{tabular}{ccc}
\hline Edad (días) & Control (kg) & Moringa $(\mathbf{k g})$ \\
\hline 7 & $4.42 \pm 0.425$ & $4.04 \pm 0.327$ \\
14 & $11.42 \pm 0.915$ & $9.13 \pm 0.721$ \\
21 & $18.00 \pm 0.740$ & $12.49 \pm 0.951$ \\
28 & $24.45 \pm 1.198$ & $16.77 \pm 1.114$ \\
35 & $30.90 \pm 1.255$ & $27.65 \pm 1.300$ \\
42 & $34.16 \pm 1.322$ & $37.11 \pm 1.625$ \\
& $123.35 \pm 7.255$ & $107.19 \pm 6.234$ \\
\hline
\end{tabular}

Se sabe que cuanto más bajo es el índice de conversión, mayor eficiencia del alimento para aumentar el peso del animal(26). En este estudio se observó que el índice de conversión fue similar solo en la segunda y tercera semana (Figura 1C). En el estudio 
realizado con $M$. stenopetala no se observaron diferencias en el índice de conversión cuando los pollos recibieron 5 y $8 \%$ de la hoja de moringa comparado con el testigo ${ }^{(4)}$. Sin embargo, con mayores niveles, el índice de conversión fue mayor en los animales tratados. Se ha reportado que una dieta con $14 \%$ de hoja de moringa generó un menor peso en pollos. Los resultados observados en el peso promedio, la ganancia en el peso y el índice de conversión afectaron el índice de productividad de los pollos con moringa. En este aspecto, el grupo testigo presentó un valor $21 \%$ mayor al grupo adicionado con la hoja de moringa (Figura 2D).

No se observaron diferencias significativas en el contenido de proteínas totales (PT) (Figura 2A). Por el contrario, el contenido de albúminas fue mayor en los pollos a los que se les dio moringa (Figura 2B). Sin embargo, en ambos casos fue menor a los valores de referencia reportados para aves $(3.1 \text { a } 5.05 \mathrm{~g} / \mathrm{dl})^{(34)}$. Los bajos niveles de globulina presentados por ambos grupos en este experimento pudieran estar asociados a problemas patológicos en hígado y riñón, por un lado, o factores intrínsecos propios de los animales bajo estudio.

Figura 2: Contenido de, A) proteínas totales (g/dl); B) albumina (g/dl) y actividad enzimática en U/L de C) alanino aminotransferasa ; D) aspartato aminotransferasa ; E) fosfatasa alcalina y F) gamma glutamil transferasa en pollos alimentados con moringa
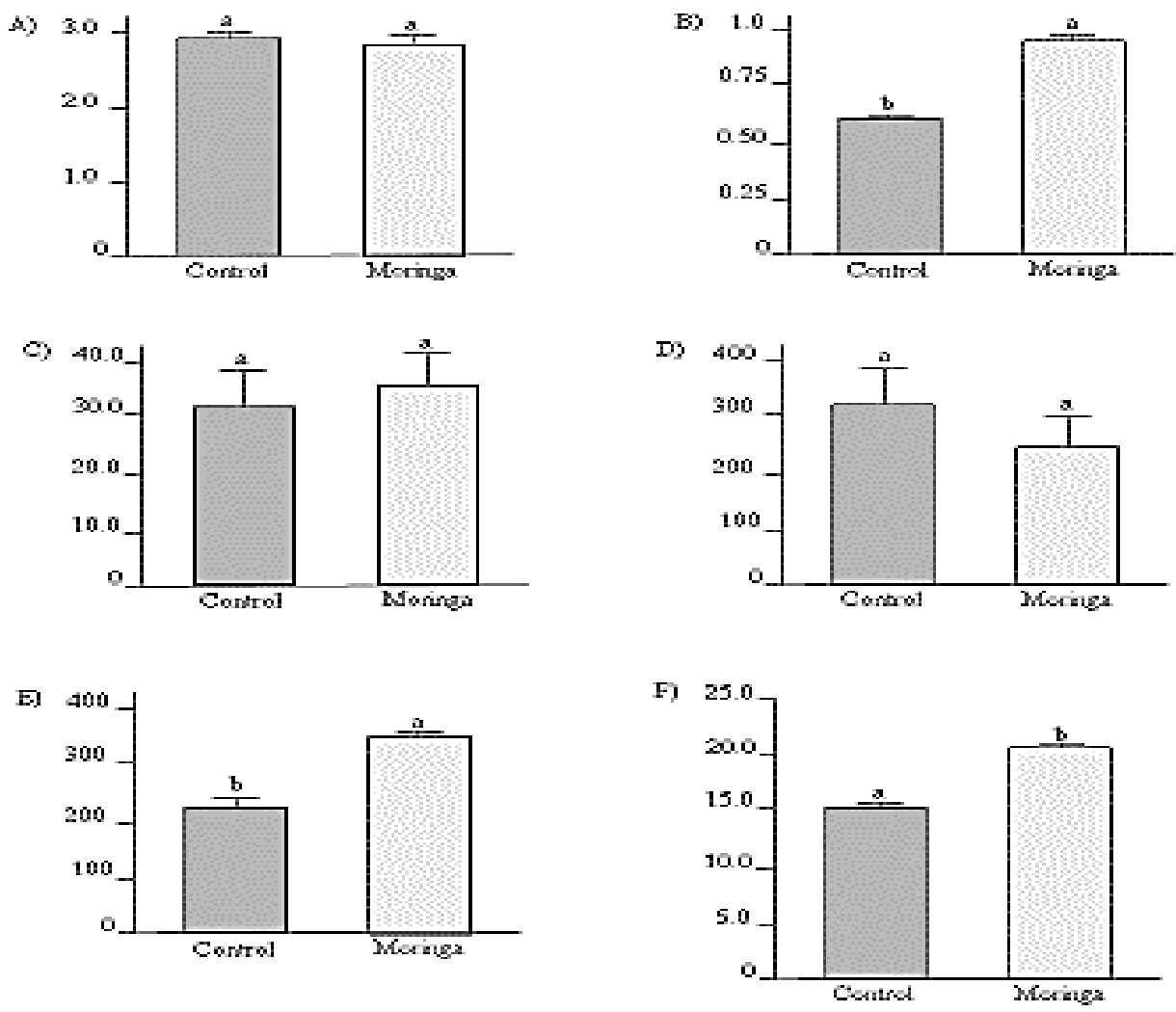

${ }^{\text {ab }}$ Literales con letras iguales no son diferentes $(P<0.05, \mathrm{n}=90)$. 
En relación con las enzimas bioquímicas evaluadas, no se observaron diferencias en las actividades enzimáticas de ALT y AST (Figura 2C y 2D). Sin embargo, el grupo alimentado con moringa alcanzó un nivel de ALT de 35.1 UI/L cercano al límite máximo de referencia para esta enzima (9.5 a $37.2 \mathrm{UI} / \mathrm{L})^{(35)}$; un valor por arriba del límite superior de la ALT sugiere daño hepático y renal ${ }^{(36)}$. Con respecto a la AST, no se observó una diferencia $(P>0.05)$. Se ha reportado que cuando existe daño hepático crónico se puede presentar ruptura de células sutiles, lo cual se manifiesta en niveles normales de la AST, pero más particularmente cuando se presenta una disminución en la actividad de esta enzima, como es el caso en el grupo de pollos alimentados con moringa (Figura 2D) ${ }^{(37)}$. Sin embargo, de manera general, valores de la actividad enzimática de AST arriba de 275 UI/L pueden estar relacionados con disturbios hepáticos o musculares; los valores de la actividad enzimática AST por encima de $800 \mathrm{UI} / \mathrm{L}$ son altamente sugestivos de daño hepático severo ${ }^{(38)}$, situación que no se presenta en el presente trabajo.

Se observaron diferencias estadísticas en la actividad enzimática de la FA y de la GGT (Figura 2E y 2F). Se ha reportado un valor de actividad enzimática de referencia de 600 $\mathrm{UI} / \mathrm{L}$ para la $\mathrm{FA}^{(4)}$ valor que no fue observado en ambos grupos de pollos. Sin embargo, el aumento en la actividad de esta enzima sugiere crecimiento óseo, osteomielitis y neoplasias $^{(38)}$; no se indica si estos problemas se presentan cuando los niveles de actividad enzimática para la FA son mayores al valor de referencia reportado. No es posible comprobar si se presentó este problema en los pollos alimentados con moringa con base a los resultados obtenidos en este estudio.

La GGT es una enzima de membrana asociada a una proteína y está relacionada con el nivel del metabolismo de los aminoácidos. Incrementos en la actividad de esta enzima son originados por inflamación, colestasis biliar e hiperplasia de los conductos biliares ${ }^{(39)}$. En el presente estudio se observó un incremento de $25 \%$ en la actividad de la GGT en el grupo alimentado con moringa (Figura 2F). Valores de GGT entre 0-10 UI/L son considerados normales ${ }^{(40)}$. Tomando como referencia los valores aquí citados, se podría concluir que ambos grupos de aves estudiados pudieran presentar una lesión hepática $\operatorname{activa}^{(41)}$.

El estudio histopatológico de la histo-arquitectura, espacios de Möll y Disse, sinusoides, conductos biliares y la relación núcleo-citoplasma, no mostraron diferencias micromorfológicas entre ambos grupos (Figura 3A y 3B); debido a que las estructuras evaluadas no presentaron congestión, inflamación, tejido cicatrizal, licuefacción, vacuolas lipídicas, picnosis, cariolisis y cariorexis. 
Figura 3: Histopatologías de hígado en pollos testigo (A), de hígado en pollos suplementados (B), de riñón en pollos control (C), de riñón, en pollos suplementados

(D)
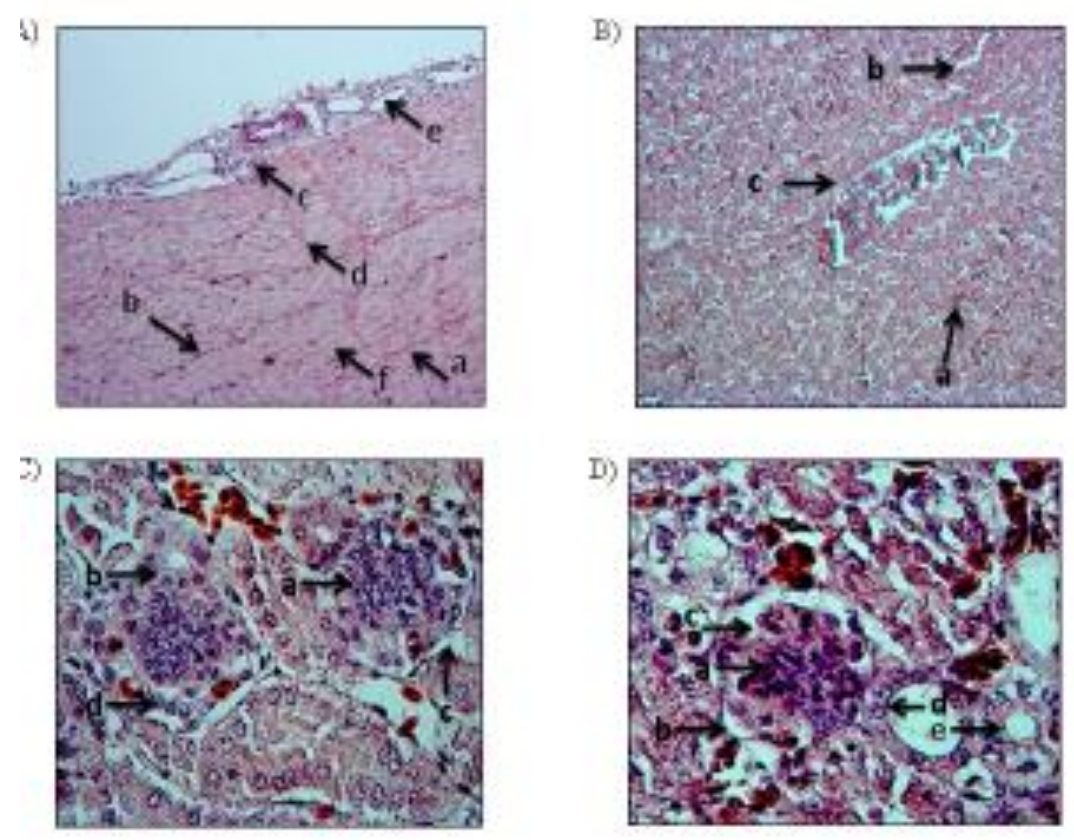

A) (a) histo-arquitectura del parénquima, (b) células basófilas, (c) espacio de Möll, (d) sinusoides, (e) conductos biliares, (f) hepatocitos.

B) (a) parénquima, (b) sinusoides y (c) vena centro lobulillar.

C) (a) glomérulo renal, (b) túbulo proximal, (c) cápsula de Bowman, (d) capa visceral del glomérulo.

D) (a) glomérulo renal, (b) espacio urinario reducido, (c) barrera de filtración, (d) mácula densa aparentemente normal, (e) túbulo proximal íntegro.

Con respecto a los estudios histopatológicos realizados en los riñones, (Figura 3C y D), en los que se evaluaron microscópicamente el glomérulo, la cápsula de Bowman, el espacio urinario, los podocitos, el polo vascular y urinario, la barrera de filtración, los túbulos proximales, la asa de Henle, los túbulos distales, el aparato yuxtaglomerular los túbulos colectores y el intersticio renal, se pudo observar que tampoco hubo diferencias micro-morfológicas entre ambos grupos; ya que las estructuras evaluadas no mostraron congestión, inflamación e integridad de sus estructuras.

Se concluye que la hoja de moringa es buena fuente de proteína y minerales. Con la adición del $10 \%$ de harina de moringa a la dieta de los pollos de engorda se disminuyeron los parámetros productivos durante el periodo que duró el estudio. A pesar de observar algunas diferencias en las proteínas y enzimas evaluadas, no se detectaron efectos tóxicos visibles, lo cual fue confirmado por los estudios histopatológicos. Los mayores niveles de la actividad de la GGT y FA en el grupo bajo tratamiento pudieron deberse a ciertos procesos inflamatorios en los conductos biliares en ambos grupos ocasionados por factores ajenos al consumo de la moringa y del alimento. Se sugiere seguir realizando estudios con niveles de complementación menor con la harina de moringa para comparar los efectos encontrados en el presente estudio y así poder definir su uso potencial. 
Un agradecimiento al CONACYT por la beca de maestría otorgada a Fuentes-Esparza Martha K., y al INIFAP por los fondos asignados al presente estudio en el marco del proyecto fiscal "Potencial agronómico, nutricional y nutracéutico de la moringa (Moringa oleífera) y usos alternativos de la hoja y la pasta de la semilla" (No. Preci 11163719248).

\section{Literatura citada:}

1. SAGARPA. Pollos, gallinas y avicultura en México 2017, www.gob.mx/sagarpa/articulos/pollos-gallinas-y-la-avicultura-en-mexico.

2. Cuca MG, Ávila EG, Fuente de energía y proteínas para la alimentación de las aves. Ciencia Vet 1978; 2:325-358.

3. Romero-Lara ML, Producción avícola a pequeña escala. Ficha 12. Colegio de Posgraduados. SAGARPA, Subsecretaria de Desarrollo Rural. https://docplayer.es/5952656-Produccion-avicola-a-pequena-escala.html

4. Melesse A, Getye Y, Berihun K, Banerjee S, Effect of feeding graded levels of Moringa stenopetala leaf meal on growth performance, carcass traits y some serum biochemical parameters of Koekoek chickens. Livest Sci 2013;157:498-505.

5. Sarwatt S. Substituting sunflower seed-cake with Moringa oleifera leaves as supplemental goat feed in Tanzania. Agrofor Syst 2002;56(3):241-247.

6. Jongrungruangchok SBS, Songsak T. Nutrients y minerals content of eleven different samples of Moringa oleifera cultivated in Thailand. J Health Res 2010;24(3):123-127.

7. Olson ME, Fahey JW, Moringa oleifera: Un árbol multiusos para las zonas tropicales secas. Rev Mex Biodiv 2011;82:1071-1082.

8. Yameogo CW, Bengaly MD, Savadogo A, Nikiema PA, Traore SA, Determination of chemical composition y nutritional values Moringa oleifera leaves. Pak J Nutr 2011;10:264-268.

9. Guzmán-Maldonado SH, Zamarripa-Colmenares A, Hernández-Duran LG, Calidad nutrimental y nutraceutica de hoja de moringa proveniente de árboles de diferente altura. REMEXA 2015;6:317-330.

10. Devaraj VC, Asad M, Prasad S, Effect of leaves y fruits of Moringla oleifera on gastric y duodenal ulcers. Pharmaceu Biol 2007;45:332-338.

11. Chumarka P, Khunawat P, Sanvarinda Y, Phornchirasilp S, Morales NP, PhivthongNgam L, et al. The in vitro and ex vivo antioxidant properties, hypolipidaemic and antitherosclerotic activities of water extract fo Moringa oleifera Lam. leaves. J Ethnoparmacol 2008;116:439-446. 
12. Sun B, Zhang Y, Ding M, Xi Q, Liu G, Li Y, et al. Effects of Moringa oleifera leaves as a substitute for alfalfa meal on nutrient digestibility, growth performance, carcass trait, meat quality, antioxidant capacity and biochemical parameters of rabbits [en prensa]. J Anim Physiol Anim Nutr 2017, doi: 10.1111/jpn.12678.

13. Babiker EE, Al Juhaimi F, Ghafoor K, Mohamed HE, Abdoun KA, Effect of partial replacement of alfalfa hay with Moringa species leaves on milk yield and composition of Najdi ewes. Trop Anim Health Prod 2016;48(7):1427-1433.

14. Mendieta-Araica B, Spörndly E, Reyes-Sánchez N, Spörndly R, Feeding Moringa oleifera fresh or ensiled to dairy cows--effects on milk yield and milk flavor. Trop Anim Health Prod 2011;43(5):1039-1047.

15. Khan I, Zaneb H, Masood S, Yousaf MS, Rehman HF, Rehman H, Effect of Moringa oleifera leaf powder supplementation on growth performance and intestinal morphology in broiler chickens. J Anim Physiol Anim Nutr (Berl)2017;(Suppl 1):114-121.

16. Ayssiwede, SB. Effects of Moringa oleifera (Lam) leaves meal incorporation in diets on growth performances, carcass characteristics y economics results of growing indigenous Senegal chickens. Pak J Nutr 2011;10(12):1132-1145.

17. Nkukwana TT, Muchenje V, Masika PJ, Pieterse E, Hoffman LC, Dzama K, Proximate composition and variation in colour, drip loss and $\mathrm{pH}$ of breast meat from broilers supplemented with Moringa oleifera leaf meal over time. Anim Prod Sci 2016;56(7):1208-1216.

18. Nkukwana TT, Muchenje V, Masika PJ, Hoffman LC, Dzama K, The effect of Moringa oleifera leaf meal supplementation on tibia strength, morphology y inorganic content of broiler chickens. South African J Anim Sci 2014; 44:228-239.

19. Liaqat S, Mahmood S, Ahmad S, Kamran Z, Koutoulis KC, Replacement of canola meal with Moringa oleifera leaf powder affects performance and immune response in broilers. J App Poultry Res 2016;25(3):352-358.

20. National Research Council. Subcommittee on Poultry Nutrition, Committee on Animal Nutrition, Board on Agriculture. National Academy Press, Washington, DC, Ninth Revised Edition. 1994.

21. AOAC. Official methods of analysis of the Association of Official Analytical Chemists International: Vitamins and other nutrients. 17th ed. Gaithersburg, USA: Hoerwitz W ed.; 2000.

22. Jones JB, Case VW. Sampling, handling, and analyzing plant tissue samples In: Westerman RL editor. Soil testing and plant analysis. Book Series 3, Soil Sci Soc Am. Madison, Wisconsin, USA. 1990:389-427.

23. Tsao R, Yang R, Young JC, Zhu H, Polyphenolic profiles in eight apple cultivars 
using high-performance liquid chromatography (HPLC). J Agric Food Chem 2003;51:6347-6353.

24. Singleton VL, Orthofer R, Lamuela-Raventos RM, Analysis of total phenols and other oxidation substrates and antioxidants by means of folin-ciocalteu reagent. Methods Enzymol 1999;299:152-178.

25. Deshpande SS, Cheryan M, Evaluation of vanillin assay for tannin analysis of dry beans. J Food Sci 1985;50:905-910.

26. Quintana JA. Avitecnia: manejo de las aves domésticas más comunes. 4ta ed. Ciudad de Mexico, Mx: Trillas; 2011.

27. Burtis CA, Ashwood ER. Clinical chemistry. Philadelphia, USA: Saunders WB; 1999.

28. McElroy DA, Prophet EB, Mills B, Arrington JB, Sobin LH. Laboratory methods. In Histotechnology. Armed Forces Institute of Pathology, American Registry of Pathology.Washington, DC: 1994.

29. Statiscal Analysis System (SAS). General Linear Models (GLM). North Caroline State Universality, Raleigh, North Caroline, USA: 2001.

30. Makkar HPS, Becker K. Nutrients and antiquality factors in different morphological parts of the Moringa oleifera tree. J Agric Food Sci 1997;128:311-322.

31. Ross B, Suplemento de nutrición del pollo de engorda. http://es.aviagen.com/assets/Tech_Center/BB_Foreign_Language_Docs/Spanish_T echDocs/RossBroilerHandbook2014-ES.pdf.

32. Nagórna-Stasiak B, Lazuga-Adamczyk A. The effect of iron on metabolism of vitamin C in chickens. Arch Vet Pol 1994;34(1-2):99-106.

33. Hassan IAG, Elzubeir EA, Tinay AH. Growth and apparent absorption of minerals in broiler chicks fed diets with low and high tannin contents. Trop Anim Health Prod 2003;35:189-196.

34. Meluzzi A, Primiceri G, Giordani R, Fabris G. Determination of blood constituents reference values in broilers. Poult Sci 1992;71:337-345.

35. Mitruka BM, Rawnsley HM. Clinical biochemical and hematological reference values in normal experimental animals. 1rst ed. New York, USA: Masson Publishing USA Inc.; 1997.

36. Landeros P, Reyes W, De Lucas E, Albarrán E, López Y, Quezada T. Evaluación de dos adsorbentes (manano oligosacáridos y clinoptilolita) en dietas de pollos de engorde contaminadas con fumonisina B1. Rev Salud Anim 2008;30:50-58.

37. Werner LL, Laboratory medicine - Avian and exotic pets. Vet Clin Pathol 
2001;30(1):2-46.

38. Thrall MA, Weise G, Allison RW, Campbell TW. Clinical chemistry of birds. In: Thrall MA editor. Veterinary hematology and clinical chemistry. 2nd ed. Philadelphia, Lippincott, USA: Wiley-Blackwell; 2004:479-492.

39. Fernandez A, Verde MT, Gascon M, Ramos J, Gomez J, Luco DF, et al. Variations of clinical biocheT1mical parameters of laying hens y broiler chickens fed aflatoxincontaining feed. Avian Pathol 1994;23:37-47.

40. Lumeij JT. Avian clinical biochemistry. In: Kaneko JJ, Harvey JW, Bruss ML editors. Clinical biochemistry of domestic animals. 5th ed. San Diego, California, USA: Academic Press; 1995:308-335.

41. Fudge AM. Avian liver and gastrointestinal testing. In: Fudge AM editor. Laboratory Medicine - Avian and Exotic Pets. 1rst ed. St. Louis, Missouri, USA: WB Saunders Co; 2000:47-55. 